\title{
Bayer Corneal Epitheliopathy Grade 1
}

National Cancer Institute

\section{Source}

National Cancer Institute. Bayer Corneal Epitheliopathy Grade 1. NCI Thesaurus. Code C128440.

Any stage of superficial punctate keratitis. 